\title{
Erratum to: Impact of Surgical Infection Society/Infectious Disease Society of America-recommended antibiotics on postoperative intra-abdominal abscess with image-guided percutaneous abscess drainage following gastrointestinal surgery
}

\author{
Yoshiki Okita • Minako Kobayashi · Toshimitsu Araki • Hiroyuki Fujikawa • Yuhki Koike • Kohei Otake • \\ Mikihiro Inoue $\cdot$ Yuji Toiyama $\cdot$ Masaki Ohi $\cdot$ Koji Tanaka $\cdot$ Yasuhiro Inoue $\cdot$ Keiichi Uchida $\cdot$ Yasuhiko Mohri $\cdot$ \\ Koichiro Yamakado $\cdot$ Masato Kusunoki
}

Published online: 25 February 2015

(C) Springer Japan 2015

\section{Erratum to: Surg Today}

DOI 10.1007/s00595-014-1047-5

In the article cited above, the names of the fifth to ninth authors appeared incorrectly. The correct list of authors is as follows.

Yoshiki Okita · Minako Kobayashi · Toshimitsu Araki · Hiroyuki Fujikawa · Yuhki Koike · Kohei Otake · Mikihiro Inoue · Yuji Toiyama $\cdot$ Masaki Ohi · Koji Tanaka · Yasuhiro Inoue - Keiichi Uchida · Yasuhiko Mohri - Koichiro Yamakado · Masato Kusunoki

The online version of the original article can be found under doi:10.1007/s00595-014-1047-5.

Y. Okita $(\bowtie) \cdot$ M. Kobayashi · T. Araki $\cdot$ H. Fujikawa $\cdot$ Y. Koike

K. Otake $\cdot$ M. Inoue $\cdot$ Y. Toiyama $\cdot$ M. Ohi $\cdot$ K. Tanaka $\cdot$ Y. Inoue

K. Uchida $\cdot$ Y. Mohri $\cdot$ M. Kusunoki

Department of Gastrointestinal and Pediatric Surgery, Mie

University Graduate School of Medicine, Tsu, Japan

e-mail: nyokkin@clin.medic.mie-u.ac.jp

K. Yamakado

Department of Radiology, Mie University Graduate School

of Medicine, 2-174 Edobashi, Tsu, Mie 514-8507, Japan 